\title{
ON SIMULTANEOUS ADDITIVE EQUATIONS, IV
}

\author{
TREVOR D. WOOLEY
}

\$1. Introduction. Recent advances in the theory of exponential sums (see, for example, [6], [7], [8], [12]) have contributed to corresponding progress in our understanding of the solubility of systems of simultaneous additive equations (see, in particular, [1], [2], [3], [4]). In a previous memoir [11] we developed a version of Vaughan's iterative method (see Vaughan [8]) suitable for the analysis of simultaneous additive equations of differing degrees, discussing in detail the solubility of simultaneous cubic and quadratic equations. The mean value estimates derived in [11] are, unfortunately, weaker than might be hoped, owing to the presence of undesirable singular solutions in certain auxiliary systems of congruences. The methods of [12] provide a flexible alternative to Vaughan's iterative method, and, as was apparent even at the time of their initial development at the opening of the present decade, such ideas provide a means of avoiding altogether the aforementioned problematic singular solutions. The systematic development of such an approach having been described recently in [15], in this paper we apply such methods to investigate the solubility of pairs of additive equations, one cubic and one quadratic, thereby improving the main conclusion of [11].

Let $s$ be a positive integer, and let $c_{i}$ and $d_{i}(1 \leqslant i \leqslant s)$ be rational integers. We consider the solubility, in rational integers $x_{i}(1 \leqslant i \leqslant s)$, of the simultaneous additive equations

$$
\begin{aligned}
& F(\mathbf{x})=c_{1} x_{1}^{3}+c_{2} x_{2}^{3}+\ldots+c_{s} x_{s}^{3}=0, \\
& G(\mathbf{x})=d_{1} x_{1}^{2}+d_{2} x_{2}^{2}+\ldots+d_{s} x_{s}^{2}=0,
\end{aligned}
$$

establishing in $\$ 5$ the following theorem.

THEOREM 1. The simultaneous equations (1.1) have a non-trivial solution in rational integers (which is to say, a solution $\mathbf{x} \in \mathbb{Z}^{s} \backslash\{0\}$ ) if the following conditions hold:

(a) the polynomial $G(\mathbf{x})$ is indefinite, and has at least 5 variables explicit; and

(b) the polynomial $F(\mathbf{x})$ has at least 7 variables explicit; and

(c) the system $F(\mathbf{x})=G(\mathbf{x})=0$ has a non-trivial real solution; and

(d) one has

(i) $s \geqslant 13$, or

(ii) $s \geqslant 12$ and at least 2 of the $c_{i}$ are zero, or

(iii) at least 6 of the $d_{i}$ are zero, or

(iv) at least 4 of the $c_{i}$ are zero.

The conclusion of Theorem 1 may be compared with [11, Theorem 1], where a similar result is established with condition (d)(i) replaced with the 
more stringent hypothesis that $s \geqslant 14$, and with the alternative condition (d)(ii) absent. We note that the system (1.1) possesses non-trivial solutions in every $p$-adic field $\mathbb{Q}_{p}$ provided only that $s \geqslant 11$ (see the main theorem of [10]), and moreover that when $p$ is a prime number with $p \equiv 1(\bmod 3)$, then whenever $s \leqslant 10$ one may exhibit examples of the shape (1.1) which possess no non-trivial $p$-adic solutions (see [10, Lemma 7.2]). It is natural to enquire to what extent the conditions (a), (b) and (c) of Theorem 1 may be relaxed. For a discussion of such issues we refer the reader to $[\mathbf{1 1}, \S 5]$; in particular, there exist systems (1.1) for which $G(\mathbf{x})$ is indefinite, and yet the equation $F(\mathbf{x})=G(\mathbf{x})=0$ possesses no non-trivial real solutions.

The proof of Theorem 1 depends for its success on a mean value estimate for smooth Weyl sums. This we establish in $\$ 2$ through the use of the efficient differencing process described in [15]. Since this estimate may be of independent interest, we record it in Theorem 2 below. We first describe some notation. When $P$ and $R$ are real numbers with $2 \leqslant R \leqslant P$, define $\mathscr{A}(P, R)$ to be the set of $R$-smooth numbers up to $P$, that is

$$
\mathscr{A}(P, R)=\{n \in \mathbb{Z} \cap[1, P]: p \mid n \text { and } p \text { prime implies that } p \leqslant R\} .
$$

Also, write $e(z)$ for $e^{2 \pi i z}$, and when $k$ is a natural number write $\mathbb{T}^{k}$ for the unit box $[0,1]^{k}$. We define the smooth Weyl sum $f(\boldsymbol{\alpha})=f\left(\alpha_{3}, \alpha_{2} ; P, R\right)$ by

$$
f(\boldsymbol{\alpha} ; P, R)=\sum_{x \in . \mathscr{\alpha}(P . R)} e\left(\alpha_{3} x^{3}+\alpha_{2} x^{2}\right),
$$

and the corresponding classical Weyl sum $F(\boldsymbol{\alpha})=F\left(\alpha_{3}, \alpha_{2} ; P\right)$ by

$$
F(\boldsymbol{\alpha} ; P)=\sum_{1 \leqslant x \leqslant P} e\left(\alpha_{3} x^{3}+\alpha_{2} x^{2}\right)
$$

THEOREM 2. Suppose that $\varepsilon$ is a positive number, and that $\eta$ is a positive number sufficiently small in terms of $\varepsilon$. Then whenever $P$ is sufficiently large in terms of $\varepsilon$ and $\eta$, one has

$$
\int_{\pi^{2}}\left|F(\boldsymbol{\alpha} ; P)^{4} f\left(\boldsymbol{\alpha} ; P, P^{\eta}\right)^{6}\right| d \boldsymbol{\alpha} \ll P^{(17 / 3)+\varepsilon,}
$$

and here the implicit constant depends at most on $\varepsilon$ and $\eta$.

We note that [11, Theorem 2] provides a similar estimate, save that the exponent $35 / 6$ is obtained in place of our new exponent $17 / 3$.

We establish Theorem 1 by applying the Hardy-Littlewood method. In broad outline, we follow the treatment described in our previous work [11] on the topic. In $\$ 2$ we provide both the mean value estimate described in Theorem 2 , together with a second new mean value estimate required to establish part (d)(ii) of Theorem 1. After negotiating some preliminary simplifications in $\$ 3$, we go on in $\$ 4$ to apply the mean value estimates of $\$ 2$ in order to provide suitable estimates on the minor arcs of the Hardy Littlewood dissection. Finally, in $\S 5$, we describe a pruning procedure on the major arcs, and thereby establish an asymptotic formula for the number of solutions of the system (1.1) inside a suitable large box. The proof of Theorem 1 follows immediately. 
Throughout, the letter $s$ will denote a positive integer, and $\varepsilon$ and $\eta$ will denote sufficiently small positive numbers. We take $P$ to be a large positive real number depending at most on $s, \varepsilon$ and $\eta$. The implicit constants in Vinogradov's well-known notation, $\ll$ and $\gg$, will depend on $s, \varepsilon$ and $\eta$, unless otherwise indicated. We adopt the following convention concerning the numbers $\varepsilon$ and $R$. Whenever $\varepsilon$ or $R$ appear in a statement, either implicitly or explicitly, we assert that for each $\varepsilon>0$, there exists a positive number $\eta=$ $\eta(\varepsilon, s)$ such that the statement holds whenever $R \leqslant P^{\eta}$. Note that the "value" of $\varepsilon$, and of $\eta$, may change from statement to statement, and hence also the dependency of implicit constants on $\varepsilon$ and $\eta$. We observe that since our methods will involve only a finite number of statements (depending at most on $s$ and $\varepsilon$ ), there is no danger of losing control of implicit constants through the successive changes implicit in our arguments.

\$2. Mean value estimates for exponential sums. In this section we establish the mean value estimates essential to our proof of Theorem 1. We begin by establishing Theorem 2 through the efficient differencing process described in $[15, \S 5]$. We first require some additional notation. Let $\theta$ be a parameter with $0<\theta \leqslant 1 / 3$ to be chosen later, and write

$$
M=P^{\theta}, \quad H=P M^{-3} \quad \text { and } \quad Q=P M^{-1} .
$$

Further, define the polynomial $\Psi_{i}(z ; h ; m)(i=2,3)$ by

$$
\Psi_{i}(z ; h ; m)=\left(z+h m^{3}\right)^{i}-z^{i},
$$

and write

$$
G(\boldsymbol{\alpha} ; m)=\sum_{1 \leqslant h \leqslant H} \sum_{1 \leqslant z \leqslant P} e\left(\alpha_{3} \Psi_{3}(z ; h ; m)+\alpha_{2} \Psi_{2}(z ; h ; m)\right) .
$$

Also, define the exponential sum $g_{m}(\boldsymbol{\alpha} ; Q, R)$ by

$$
g_{m}(\boldsymbol{\alpha} ; Q, R)=\sum_{x \in, \mathscr{\alpha}(Q, R)} e\left(\alpha_{3}(m x)^{3}+\alpha_{2}(m x)^{2}\right) .
$$

Finally, with $F(\boldsymbol{\alpha} ; P)$ and $f(\boldsymbol{\alpha} ; P, R)$ as in (1.2) and (1.3), define the mean values

$$
\begin{aligned}
\mathscr{H}(P, R) & =\sum_{M<m \leqslant M R} \int_{\mathbb{T}^{2}}\left|G(\boldsymbol{\alpha} ; m)^{2} g_{m}(\boldsymbol{\alpha} ; Q, R)^{6}\right| d \boldsymbol{\alpha}, \\
U(P) & =\int_{\mathbb{J}^{2}}|F(\boldsymbol{\alpha} ; P)|^{6} d \boldsymbol{\alpha},
\end{aligned}
$$

and

$$
\mathscr{P}(P, R)=\int_{\mathbb{J}^{2}}\left|F(\boldsymbol{\alpha} ; P)^{4} f(\boldsymbol{\alpha} ; P, R)^{6}\right| d \boldsymbol{\alpha} .
$$


Before recalling a special case of $[\mathbf{1 5}$, Lemma 5.1], it is convenient to record an estimate for $U(P)$.

LeMma 2.1. One has $U(P) \ll P^{3+\varepsilon}$.

Proof. On considering the underlying diophantine equations, the conclusion of the lemma is immediate from [11, Theorem 4.1].

Although we will not require estimates sharper than Lemma 2.1 in what follows, we note that the asymptotic formula

$$
U(P)=6 P^{3}+O\left(P^{(7 / 3)+\varepsilon}\right)
$$

has recently been established (see [14, Theorem 1]).

Lemma 2.2. Suppose that $0<\theta \leqslant 1 / 3$. Then

$$
\mathscr{S}(P, R) \ll P^{\varepsilon} M^{6}\left(P^{2} M U(Q)+\mathscr{M}(P, R)\right) .
$$

Proof. The conclusion of the lemma follows from [15, Lemma 5.1] on taking $s=3, r=2, \mathbf{k}=(3,2), k=3$, and noting that, on considering the underlying diophantine equations, one has

$$
\int_{\mathbb{U}^{2}}|f(\boldsymbol{\alpha} ; Q, R)|^{6} d \boldsymbol{\alpha} \ll U(Q)
$$

In order to exploit Lemma 2.2 we employ estimates for $\mathscr{M}(P, R)$ contained, in all essentials, in the proof of [11, Lemma 4.3].

LeMma 2.3. One has $\mathscr{S}(P, R) \ll P^{(17 / 3)+\varepsilon}$.

Proof. We first estimate $\mathscr{M}(P, R)$. Observe that by Cauchy's inequality, it follows from (2.3) that

$$
|G(\boldsymbol{\alpha} ; m)|^{2} \leqslant H \sum_{1 \leqslant h \leqslant H}\left|\sum_{1 \leqslant z \leqslant P} e\left(\alpha_{3} \Psi_{3}(z ; h ; m)+\alpha_{2} \Psi_{2}(z ; h ; m)\right)\right|^{2},
$$

and hence, on considering the underlying diophantine equations, we deduce from (2.5) together with (2.2) and (2.4) that

$$
\mathscr{M}(P, R) \ll H V(P, R),
$$

where $V(P, R)$ denotes the number of solutions of the simultaneous diophantine equations

$$
\begin{aligned}
3 h\left(z_{1}^{2}-z_{2}^{2}\right)+3 h^{2} m^{3}\left(z_{1}-z_{2}\right) & =\sum_{i=1}^{3}\left(u_{i}^{3}-v_{i}^{3}\right), \\
2 h m\left(z_{1}-z_{2}\right) & =\sum_{i=1}^{3}\left(u_{i}^{2}-v_{i}^{2}\right),
\end{aligned}
$$


with

$$
1 \leqslant z_{1}, z_{2} \leqslant P, \quad 1 \leqslant h \leqslant H, \quad M<m \leqslant M R
$$

and

$$
u_{i}, v_{i} \in \mathscr{A}(Q, R) \quad(1 \leqslant i \leqslant 3)
$$

On considering the underlying diophantine equations, the number of solutions, $V_{0}$, of the system (2.8) counted by $V(P, R)$ for which $z_{1}=z_{2}$ plainly satisfies

$$
V_{0} \ll P M R H U(Q)
$$

Suppose then that $h, m, \mathbf{z}, \mathbf{u}, \mathbf{v}$ is a solution of $(2.8)$ counted by $V(P, R)$ with $z_{1} \neq z_{2}$. Writing

$$
x=z_{1}-z_{2} \text { and } y=z_{1}+z_{2}+h m^{3},
$$

and eliminating $x$ between the two equations of (2.8), we obtain

$$
\sum_{i=1}^{3}\left(\psi\left(u_{i} ; m, y\right)-\psi\left(v_{i} ; m, y\right)\right)=0,
$$

where $\psi(z ; m, y)$ is the polynomial defined by

$$
\psi(z ; m, y)=2 m z^{3}-3 y z^{2} .
$$

Since, by hypothesis, we have $z_{1} \neq z_{2}$, it follows by applying an elementary divisor function estimate that given $\mathbf{u}, \mathbf{v}, m$ and $y$, there are at most $O\left(P^{\varepsilon}\right)$ solutions in $h$ and $x=z_{1}-z_{2}$ to the system (2.8). Moreover, given $h, m, x$ and $y$, one may determine $z_{1}$ and $z_{2}$ from the equations (2.11). We conclude, therefore, that the number of solutions, $V_{1}$, of the system (2.8) counted by $V(P, R)$ for which $z_{1} \neq z_{2}$ satisfies

$$
V_{1} \ll P^{\varepsilon} \sum_{M<m \leqslant M R} \sum_{1 \leqslant y \leqslant 3 P R^{3}} N(Q ; m, y),
$$

where $N(Q ; m, y)$ denotes the number of solutions of the diophantine equation (2.12) with $\mathbf{u}, \mathbf{v}$ satisfying (2.9). But it follows from [5, Theorem 4], as in the argument following the proof of [11, Lemma 4.3], that

$$
N(Q ; m, y) \ll Q^{(7 / 2)+\varepsilon},
$$

whence, on collecting together (2.7), (2.10) and (2.13), we conclude that

$$
\mathscr{M}(P, R) \ll H\left(V_{0}+V_{1}\right) \ll P M R H^{2} U(Q)+P^{1+\varepsilon} M H Q^{7 / 2} .
$$

In order to complete the proof of the lemma, we put $\theta=1 / 6$, and substitute the upper bounds of Lemma 2.1 and (2.14) into the conclusion of Lemma 2.2. Thus, on recalling (2.1) we have

$$
\mathscr{P}(P, R) \ll P^{\varepsilon} M^{6}\left(P^{2} M Q^{3}+P M H^{2} Q^{3}+P M H Q^{7 / 2}\right) \ll P^{(17 / 3)+\varepsilon} .
$$

Bearing in mind the notational devices described in the final paragraph of the Introduction, the conclusion of Theorem 2 is immediate from Lemma 2.3. 
Before describing a second mean value estimate which we require in order to negotiate case (d)(ii) of Theorem 1, we require some additional notation. Let $a, b$ and $c$ be non-zero integers, and when $\left(\alpha_{3}, \alpha_{2}\right) \in[0,1]^{2}$, write

$$
\widetilde{f}(\boldsymbol{\alpha} ; P, R)=\sum_{x \in \mathcal{\alpha}(P, R)} e\left(a \alpha_{3} x^{3}+b \alpha_{2} x^{2}\right) \quad \text { and } \quad \tilde{H}\left(\alpha_{2} ; P\right)=\sum_{1 \leqslant y \leqslant P} e\left(c \alpha_{2} y^{2}\right)
$$

Lemma 2.4. One has

$$
\int_{J^{2}}\left|\tilde{f}(\boldsymbol{\alpha} ; P, R)^{8} \tilde{H}\left(\alpha_{2} ; P\right)^{2}\right| d \boldsymbol{\alpha} \ll P^{(16 / 3)+\varepsilon} .
$$

Proof. By considering the underlying diophantine equation, one finds that the integral on the left-hand side of $(2.15)$ is bounded above by the number, $W(P, R)$, of solutions of the simultaneous diophantine equations

$$
\begin{aligned}
& \sum_{i=1}^{4} a\left(x_{i}^{3}-y_{i}^{3}\right)=0, \\
& \sum_{i=1}^{4} b\left(x_{i}^{2}-y_{i}^{2}\right)=c\left(u^{2}-v^{2}\right),
\end{aligned}
$$

with

$$
x_{i}, y_{i} \in \mathscr{A}(P, R) \quad(1 \leqslant i \leqslant 4) \quad \text { and } \quad 1 \leqslant u, v \leqslant P .
$$

By Hua's Lemma (see, for example, [9, Lemma 2.5]), the number of possible choices for $\mathbf{x}, \mathbf{y}$ satisfying (2.16) and (2.17) is $O\left(P^{5+\varepsilon}\right)$. In particular, the number of such choices with $\sum_{i=1}^{4} b\left(x_{i}^{2}-y_{i}^{2}\right)$ non-zero is $O\left(P^{5+\varepsilon}\right)$, and for any such choice of $\mathbf{x}, \mathbf{y}$, on applying an elementary estimate for the divisor function, it follows from (2.16) that the number of possible choices for $u$ and $v$ is $O\left(P^{\varepsilon}\right)$. Consequently the number, $W_{1}$, of solutions of $(2.16)$ counted by $W(P, R)$ with $u \neq v$ satisfies

$$
W_{1} \ll P^{5+\varepsilon} \text {. }
$$

On the other hand, on considering the underlying diophantine equations one finds that the number, $W_{0}$, of solutions of (2.16) counted by $W(P, R)$ with $u=v$ satisfies

$$
W_{0} \ll P \int_{\mathbb{U}^{2}}|\tilde{f}(\boldsymbol{\alpha} ; P, R)|^{8} d \boldsymbol{\alpha} .
$$

Consequently, on making a change of variables, applying Schwarz's inequality and considering the underlying diophantine equations, we deduce that

$$
W_{0} \ll P\left(\int_{\sigma^{2}}|F(\boldsymbol{\alpha} ; P)|^{6} d \boldsymbol{\alpha}\right)^{1 / 2}\left(\int_{I^{2}}\left|F(\boldsymbol{\alpha} ; P)^{4} f(\boldsymbol{\alpha} ; P, R)^{6}\right| d \boldsymbol{\alpha}\right)^{1 / 2} .
$$

Thus we may conclude from Lemma 2.1 and Theorem 2 that

$$
W_{0} \ll P\left(P^{3+\varepsilon}\right)^{1 / 2}\left(P^{(17 / 3)+\varepsilon}\right)^{1 / 2}=P^{(16 / 3)+\varepsilon},
$$


whence by $(2.18)$ we have

$$
W(P, R)=W_{0}+W_{1} \ll P^{(16 / 3)+\varepsilon} .
$$

The lemma now follows immediately.

§3. Preliminary simplifications. Our application of the Hardy-Littlewood method in the remainder of the paper will be much simplified by making some preliminary observations. Fortunately, essentially all of the necessary work is presented in $[11, \$ 6]$. We consider henceforth the system (1.1), and start by showing that the conditions of Theorem 1 permit us to assume that the equations (1.1) have non-singular real solutions, and non-singular $p$-adic solutions for each prime $p$, whence in our application of the circle method we can expect both the singular series and singular integral to be non-zero.

Lemma 3.1. Suppose that the conditions (a), (b) and (c) of Theorem 1 hold for the equations (1.1). Then one of the following holds:

(i) the equations (1.1) have a non-trivial rational solution; or

(ii) the equations (1.1) have a real solution $\boldsymbol{\eta}=\left(\eta_{1}, \ldots, \eta_{s}\right)$ with the property that no $\eta_{i}$ is zero, and for which, locally, there is an (s-2)-dimensional subspace $\mathscr{S}$ of positive $(s-2)$-volume in the neighbourhood of $\boldsymbol{\eta}$ on which $F=G=0$.

Proof. The conclusion of the lemma follows from [11, Lemmata 6.1 and $6.2]$.

Let $M_{n}(q)$ denote the number of solutions of the simultaneous congruences

$$
\begin{aligned}
& c_{1} m_{1}^{3}+\ldots+c_{s} m_{s}^{3} \equiv 0(\bmod q), \\
& d_{1} m_{1}^{2}+\ldots+d_{s} m_{s}^{2} \equiv 0(\bmod q),
\end{aligned}
$$

with $1 \leqslant m_{i} \leqslant q \quad(1 \leqslant i \leqslant s)$.

Lemma 3.2. Suppose that the conditions (a), (b) and (c) of Theorem 1 hold for the equations (1.1), and that $s \geqslant 11$. Then one of the following holds:

(i) the equations (1.1) have a non-trivial rational solution; or

(ii) for each rational prime $p$ there is a number $u=u(p)<\infty$ such that for all $t \geqslant u$, one has $M_{n}\left(p^{t}\right) \geqslant p^{(t-u)(s-2)}$.

Proof. This is [11, Lemma 6.7].

It is also possible to dispose simply of systems in which there are many vanishing coefficients.

Lemma 3.3. Suppose that the conditions (a), (b) and (c) of Theorem 1 hold for the equations (1.1), and that $s \geqslant 11$. Then the equations (1.1) have a 
non-trivial solution in rational integers if either of the following conditions hold:

(i) at least 6 of the $d_{i}$ are zero; or

(ii) at least 4 of the $c_{i}$ are zero.

Proof. The conclusions follow in the cases (i) and (ii) respectively from [11, Lemmata 6.3 and 6.5].

In view of the conclusions of Lemma 3.3, we may assume henceforth in our proof of Theorem 1 that $s \geqslant 13$, or else that $s \geqslant 12$ and at least 2 of the $c_{i}$ are zero, and moreover that the number of zero $d_{i}$ is at most 5 , and the number of zero $c_{i}$ is at most 3 . Thus there are at least $s-8$ values of $i$ for which both $c_{i}$ and $d_{i}$ are non-zero. Also, if $c_{i}=d_{i}=0$ for any $i$, then the system (1.1) plainly possesses a non-trivial rational solution, and so we may suppose that $c_{i}=d_{i}=$ 0 for no $i$. Suppose that for $1 \leqslant i \leqslant s$ precisely $m$ of the $d_{i}$ are zero, precisely $n$ of the $c_{i}$ are zero, and let $h=10-m-n$. Then we may rearrange the variables with indices $1, \ldots, s$ so that $d_{i}=0$ for $i=1, \ldots, m$, so that $c_{i} \neq 0$ and $d_{i} \neq 0$ for $i=m+1, \ldots, s-n$, and so that $c_{i}=0$ for $i=s-n+1, \ldots, s$.

By the homogeneity of the system (1.1), together with the conclusions of Lemma 3.1, we may suppose that the equations (1.1) have a non-singular real solution $\left(\eta_{1}, \ldots, \eta_{s}\right)$ such that $0<\left|\eta_{i}\right|<2 \quad(1 \leqslant i \leqslant s)$. Moreover, since whenever necessary the $c_{i}$ can be replaced by $-c_{i}$ by interchanging $x_{i}$ and $-x_{i}$, we may suppose without loss that in fact $\eta_{i}>0 \quad(1 \leqslant i \leqslant s)$. Furthermore, in view of Lemma 3.2, we may suppose that for every rational prime $p$ there is a $u=u(p)<\infty$ such that for all $t \geqslant u$ one has $M_{n}\left(p^{t}\right) \geqslant p^{(t-u)(s-2)}$.

Our initial simplifications complete, we now record some notation to assist in our later deliberations. Let $P$ be large (in terms of $\varepsilon, \mathbf{c}, \mathbf{d}, \boldsymbol{\eta}$ ) and let $\alpha_{i}$ $(i=2,3)$ be real variables. Also, let $\delta=10^{-2}$, and write

$$
t_{2}=\max _{1 \leqslant i \leqslant s}\left|d_{i}\right| \quad \text { and } \quad t_{3}=\max _{1 \leqslant i \leqslant s}\left|c_{i}\right| .
$$

We take $\eta$ to be a positive number sufficiently small in terms of $\varepsilon$, in the context of Theorem 2, and also small enough in the context of $[\mathbf{8}$, Theorem 4.4]. When $1 \leqslant i \leqslant s$, write

$$
\begin{aligned}
& \xi_{i}=\frac{1}{2} \eta_{i}, \quad \zeta_{i}=2 \eta_{i}, \\
& F_{i}(\boldsymbol{\alpha})=F_{i}\left(\alpha_{3}, \alpha_{2}\right)=\sum_{\zeta_{i} P<x \leqslant \zeta_{i} P} e\left(c_{i} \alpha_{3} x^{3}+d_{i} \alpha_{2} x^{2}\right), \\
& f_{i}(\boldsymbol{\alpha})=f_{i}\left(\alpha_{3}, \alpha_{2}\right)=\sum_{\substack{\xi_{i} P<x \leqslant \zeta_{i} P \\
x \in, \mathcal{\alpha}(P, R)}} e\left(c_{i} \alpha_{3} x^{3}+d_{i} \alpha_{2} x^{2}\right) .
\end{aligned}
$$

When we wish to stress the presence of zero coefficients, we write

$$
g_{i}(\alpha)=f_{i}(\alpha, 0) \text { and } H_{i}(\beta)=F_{i}(0, \beta) .
$$

Our aim is to estimate the number, $R(P)$, of solutions of the diophantine system (1.1) in rational integers $x_{i}$ which satisfy

$$
\xi_{i} P<x_{i} \leqslant \zeta_{i} P \quad \text { and } \quad x_{i} \in \mathscr{A}(P, R) \quad(1 \leqslant i \leqslant 10-n),
$$


and

$$
\xi_{i} P<x_{i} \leqslant \zeta_{i} P \quad(11-n \leqslant i \leqslant s) .
$$

We will show that $R(P) \rightarrow \infty$ as $P \rightarrow \infty$ using a variant of the Hardy-Littlewood method described in [11, $\S \$ 7-10]$, thereby completing the proof of Theorem 1. Let

$$
Q_{i}=18 t_{i} P^{i-1} \quad(i=2,3), \quad \mathscr{U}_{2}^{*}=\left(Q_{2}^{-1}, 1+Q_{2}^{-1}\right) \times\left(Q_{3}^{-1}, 1+Q_{3}^{-1}\right) .
$$

Then by orthogonality, one has

$$
R(P)=\int_{\mathscr{H} \frac{*}{2}} \mathscr{F}(\boldsymbol{\alpha}) \mathscr{G}(\boldsymbol{\alpha}) \mathscr{H}(\boldsymbol{\alpha}) d \boldsymbol{\alpha},
$$

where

$$
\begin{gathered}
\mathscr{F}(\boldsymbol{\alpha})=\prod_{i=1}^{10-n} f_{i}(\boldsymbol{\alpha}), \quad \mathscr{H}(\boldsymbol{\alpha})=\prod_{i=11-n}^{s-n} F_{i}(\boldsymbol{\alpha}), \\
\mathscr{G}(\boldsymbol{\alpha})=\prod_{i=s-n+1}^{s} F_{i}(\boldsymbol{\alpha}) .
\end{gathered}
$$

The open square $\mathscr{U}_{2}^{*}$ is dissected as follows. When $\left(a_{2}, a_{3}, q\right)=1$ and $1 \leqslant a_{i} \leqslant q \leqslant P(i=2,3)$, we denote a typical major arc by

$$
\mathfrak{M}(q, \mathbf{a})=\mathfrak{M}\left(q, a_{2}, a_{3}\right)=\left\{\boldsymbol{\alpha} \in \mathscr{U}_{2}^{*}:\left|q \alpha_{i}-a_{i}\right|<Q_{i}^{-1}(i=2,3)\right\} .
$$

It may be easily verified that the $\mathfrak{M}(q, a)$ are disjoint. Let $\mathfrak{M}$ denote the union of the $\mathfrak{M}(q, \mathbf{a})$ with $1 \leqslant a_{i} \leqslant q \leqslant P \quad(i=2,3)$ and $\left(a_{2}, a_{3}, q\right)=1$, and denote by $m$ the minor arcs $m=\mathscr{U}_{2}^{*} \backslash \mathfrak{M}$. We will make use of a pruning procedure, and to this end, when $W$ is a parameter with $2 \leqslant W \leqslant P^{1 / 2}$, we define the pruned major $\operatorname{arc} \mathfrak{N}(q, \mathbf{a})$ by

$$
\mathfrak{N}(q, \mathbf{a})=\left\{\boldsymbol{\alpha} \in \mathscr{U}_{2}^{*}:\left|q \alpha_{i}-a_{i}\right|<W P^{-1} Q_{i}^{-1}(i=2,3)\right\},
$$

and take $\mathfrak{N}$ to be the union of the $\mathfrak{N}(q, \mathbf{a})$ with $1 \leqslant a_{i} \leqslant q \leqslant W(i=2,3)$ and $\left(a_{2}, a_{3}, q\right)=1$. Plainly, when $\mathfrak{N}(q, \mathbf{a}) \subseteq \mathfrak{N}$ one has $\mathfrak{N}(q, \mathbf{a}) \subseteq \mathfrak{M}(q, \mathbf{a})$, and thus $\mathfrak{N} \subseteq \mathfrak{M}$. Finally, we define $n=\mathscr{U}_{2}^{*} \backslash \mathfrak{N}$, so that $n=(\mathfrak{M} \backslash \mathfrak{N}) \cup \mathrm{m}$.

In the remainder of this paper, implicit constants in the notations of Landau and Vinogradov will depend at most on $s, \varepsilon, \eta, \mathbf{c}$, d and $\eta$, unless stated otherwise.

\$4. The minor arcs. Equipped with the new mean value estimates of $\$ 2$, our treatment of the minor arcs is a routine modification of the argument described in $[11, \S 7]$. We begin by recalling some mixed mean value estimates from $[11, \S 7]$, and also extract upper bounds from Theorem 2 and Lemma 2.4 in a convenient form. 
LeммA 4.1. Suppose that $m+1 \leqslant i \leqslant m+h, 1 \leqslant j \leqslant m$ and $s-n+1 \leqslant k \leqslant s$. Then we have:
(i) $\int_{\mathscr{U}_{2}^{*}}\left|f_{i}(\boldsymbol{\alpha})^{4} g_{j}\left(\alpha_{3}\right)^{6}\right| d \boldsymbol{\alpha} \ll P^{(21 / 4)+\varepsilon}$,
(ii) $\int_{\mathscr{U}_{2}^{*}}\left|f_{i}(\boldsymbol{\alpha})^{6} H_{k}\left(\alpha_{2}\right)^{4}\right| d \boldsymbol{\alpha} \ll P^{(21 / 4)+\varepsilon}$,
(iii) $\int_{\mathscr{Z}_{2}^{*}}\left|g_{j}\left(\alpha_{3}\right)^{6} H_{k}\left(\alpha_{2}\right)^{4}\right| d \alpha \ll P^{(21 / 4)+\varepsilon}$,
(iv) $\int_{\alpha_{2}^{*}}\left|f_{i}(\boldsymbol{\alpha})\right|^{10} d \boldsymbol{\alpha} \ll P^{(17 / 3)+\varepsilon}$,
(v) $\int_{\mathbb{U}_{2}^{*}}^{*}\left|f_{i}(\boldsymbol{\alpha})^{8} H_{k}\left(\alpha_{2}\right)^{2}\right| d \boldsymbol{\alpha} \ll P^{(16 / 3)+\varepsilon}$.

Proof. Cases (i), (ii) and (iii) of the lemma are simply the corresponding parts of [11, Lemma 7.2]. For parts (iv) and (v) we merely consider the underlying diophantine equations, and discover that the required estimates are immediate consequences of Theorem 2 and Lemma 2.4, respectively.

We are now equipped to deal with the contribution of the minor arcs.

LEMMA 4.2. We have

$$
\int_{\mathrm{m}}|\mathscr{F}(\boldsymbol{\alpha}) \mathscr{G}(\boldsymbol{\alpha}) \mathscr{H}(\boldsymbol{\alpha})| d \boldsymbol{\alpha} \ll P^{s-5-\delta} .
$$

Proof. By applying the elementary inequality

$$
\left|z_{1} z_{2} \ldots z_{n}\right| \leqslant\left|z_{1}\right|^{n}+\left|z_{2}\right|^{n}+\ldots+\left|z_{n}\right|^{n}
$$

to (3.2), we deduce that for some integers $I, J, K$ satisfying

$$
m+1 \leqslant I \leqslant m+h, \quad 1 \leqslant J \leqslant m \quad \text { and } \quad s-n+1 \leqslant K \leqslant s,
$$

one has

$$
|\mathscr{F}(\boldsymbol{\alpha}) \mathscr{G}(\boldsymbol{\alpha})| \ll\left|f_{I}(\boldsymbol{\alpha})^{h} g_{J}\left(\alpha_{3}\right)^{m} H_{K}\left(\alpha_{2}\right)^{n}\right|
$$

For the sake of concision, write $f^{h} g^{m} H^{n}$ for $\left|f_{I}(\boldsymbol{\alpha})^{h} g_{J}\left(\alpha_{3}\right)^{m} H_{K}\left(\alpha_{2}\right)^{n}\right|$. Then by repeated use of the inequality (4.1), and bearing in mind that $m \leqslant 5$ and $n \leqslant 3$, we obtain (as in the proof of [11, Lemma 7.3])

$$
f^{h} g^{m} H^{n} \ll f^{10}+f^{6} H^{4}+f^{4} g^{6}+g^{6} H^{4}
$$

Moreover, when $n \geqslant 2$ one obtains

$$
f^{h} g^{m} H^{n} \ll f^{6-m} g^{m} H^{4}+f^{8-m} g^{m} H^{2} \ll f^{6} H^{4}+g^{6} H^{4}+f^{3} g^{5} H^{2}+f^{8} H^{2},
$$

so that in view of the decomposition

$$
f^{3} g^{5} H^{2}=\left(f^{4} g^{6}\right)^{5 / 12}\left(g^{6} H^{4}\right)^{5 / 12}\left(f^{8} H^{2}\right)^{1 / 6},
$$

we have

$$
f^{h} g^{m} H^{n} \ll f^{6} H^{4}+g^{6} H^{4}+f^{8} H^{2}+f^{4} g^{6} .
$$


Thus it follows from (4.2), (4.3), (4.4) and Lemma 4.1 that

$$
\int_{\|^{*}}|\mathscr{F}(\boldsymbol{\alpha}) \mathscr{G}(\boldsymbol{\alpha})| d \boldsymbol{\alpha} \ll P^{5+\Delta+\varepsilon},
$$

where $\Delta=1 / 3$ when $n \geqslant 2$, and otherwise $\Delta=2 / 3$.

Next we observe that when $11-n \leqslant i \leqslant s-n$, one has $c_{i} \neq 0$ and $d_{i} \neq 0$, and hence the argument of [11, Lemma 7.4] shows that

$$
\sup _{\boldsymbol{\alpha} \in \mathrm{m}}|\mathscr{H}(\boldsymbol{\alpha})| \ll\left(P^{(3 / 4)+\varepsilon}\right)^{s-10} .
$$

Thus, by (4.5) one has

$$
\int_{\mathrm{m}}|\mathscr{F}(\boldsymbol{\alpha}) \mathscr{G}(\boldsymbol{\alpha}) \mathscr{H}(\boldsymbol{\alpha})| d \boldsymbol{\alpha} \ll \sup _{\boldsymbol{\alpha} \in \mathfrak{m}}|\mathscr{H}(\boldsymbol{\alpha})| \int_{\mathscr{H}^{\frac{*}{2}}}|\mathscr{F}(\boldsymbol{\alpha}) \mathscr{G}(\boldsymbol{\alpha})| d \boldsymbol{\alpha} \ll P^{s-5-\tau},
$$

where $\tau=-\Delta+(s-10) / 4-\varepsilon$. Consequently, when $n \geqslant 2$ and $s \geqslant 12$, or otherwise when $s \geqslant 13$, one has $\tau>\delta$. This completes the proof of the lemma.

\$5. The major arcs. In order to successfully estimate the contribution of the major arcs, we employ a pruning procedure which, by now, is standard in applications of the Hardy-Littlewood method involving the use of smooth Weyl sums. We first record some mean value estimates crucial to our pruning argument.

Lemma 5.1. Suppose that $m+1 \leqslant i \leqslant m+h, 1 \leqslant j \leqslant m$ and $s-n+1 \leqslant k \leqslant s$. Then whenever $t>4$ one has:

(i) $\int_{\not 13}\left|f_{i}(\boldsymbol{\alpha})^{8} H_{k}\left(\alpha_{2}\right)^{t}\right| d \boldsymbol{\alpha} \ll P^{i+3}$,

(ii) $\int_{1 / 2}\left|g_{j}\left(\alpha_{3}\right)^{8} H_{k}\left(\alpha_{2}\right)^{t}\right| d \alpha \ll P^{t+3}$,

and also one has

(iii) $\int_{1 / 2}\left|f_{i}(\boldsymbol{\alpha})^{6} g_{j}\left(\alpha_{3}\right)^{8}\right| d \boldsymbol{\alpha} \ll P^{9}$,

(iv) $\int_{\text {"15 }}\left|f_{i}(\boldsymbol{\alpha})\right|^{14} d \boldsymbol{\alpha} \ll P^{9}$.

Proof. When $t>4$, it follows as a simple application of the Hardy-Littlewood method that whenever $I$ is an index for which $d_{l}$ is non-zero, then one has

$$
\int_{0}^{1}\left|H_{I}(\alpha)\right|^{t} d \alpha \ll P^{t-2}
$$

Thus the conclusions of parts (i) and (ii) of the lemma follow by applying the argument of the proof of parts (i) and (ii) of [11, Lemma 9.1], substituting (5.1) for [11, inequality (9.1)]. Finally, the conclusions of parts (iii) and (iv) are simply Lemmata 9.1(iii) and 9.4, respectively, of [11]. 
Lemma 5.2. (a) Suppose that both $c_{i}$ and $d_{i}$ are non-zero, and that $t>9$. Then we have

$$
\int_{\mathfrak{M}}\left|F_{i}(\boldsymbol{\alpha})\right|^{t} d \boldsymbol{\alpha} \ll P^{t-5}
$$

and for some $\sigma>0$,

$$
\int_{\mathfrak{M} \backslash \mathfrak{M}}\left|F_{i}(\boldsymbol{\alpha})\right|^{t} d \boldsymbol{\alpha} \ll W^{-\sigma} P^{t-5}
$$

(b) Suppose that $c_{i}, d_{i}$ and $d_{j}$ are non-zero, and that $t>6$ and $u>4$. Then we have

$$
\int_{\mathfrak{M}}\left|F_{i}(\boldsymbol{\alpha})^{t} H_{j}\left(\alpha_{2}\right)^{u}\right| d \alpha \ll P^{t+u-5},
$$

and for some $\sigma>0$,

$$
\int_{\mathfrak{M} \backslash \mathfrak{M}}\left|F_{i}(\boldsymbol{\alpha})^{t} H_{j}\left(\alpha_{2}\right)^{u}\right| d \boldsymbol{\alpha} \ll W^{-\sigma} P^{t+u-5} .
$$

Proof. Part (a) of the lemma is [11, Lemma 9.2]. Part (b), meanwhile, follows by applying the argument of the proof of [11, Lemma 9.3], and substituting the upper bound (5.1) for [11, inequality (9.1)].

We are now ready to perform our hard pruning procedure.

LeмmA 5.3. Suppose that $s \geqslant 13$, or that $n \geqslant 2$ and $s \geqslant 12$. Then

$$
\int_{\mathscr{H}_{2}^{*}}|\mathscr{F}(\boldsymbol{\alpha}) \mathscr{H}(\boldsymbol{\alpha}) \mathscr{H}(\boldsymbol{\alpha})| d \boldsymbol{\alpha} \ll P^{s-5},
$$

and there is a positive number $\sigma$ such that

$$
\int_{n}|\mathscr{F}(\boldsymbol{\alpha}) \mathscr{G}(\boldsymbol{\alpha}) \mathscr{H}(\boldsymbol{\alpha})| d \boldsymbol{\alpha} \ll W^{-\sigma} P^{s-5} .
$$

Proof. Write $\mathscr{M}$ for either $\mathfrak{M}$ or $\mathfrak{M} \backslash \mathfrak{M}$, and let $Y=1$ if $\mathscr{M}=\mathfrak{M}$, and $Y=$ $W$ if $\mathscr{M}=\mathfrak{M} \backslash \mathfrak{N}$. Then by using the inequality (4.1) in the same way as in the proof of Lemma 4.2, one has

$$
\int_{\mathscr{M}}|\mathscr{F}(\boldsymbol{\alpha}) \mathscr{G}(\boldsymbol{\alpha}) \mathscr{H}(\boldsymbol{\alpha})| d \boldsymbol{\alpha} \ll \int_{\mathscr{M}} f^{h} g^{m} H^{n} F^{s-10} d \boldsymbol{\alpha}
$$

for some indices $I, J, K, L$, where here we have abbreviated $\left|f_{I}(\boldsymbol{\alpha})\right|$ to $f,\left|g_{J}\left(\alpha_{3}\right)\right|$ to $g,\left|H_{K}\left(\alpha_{2}\right)\right|$ to $H$, and $\left|F_{L}(\boldsymbol{\alpha})\right|$ to $F$. Recall that $m \leqslant 5, n \leqslant 3, h+m+n=10$, 
and either $n \geqslant 2$ and $s \geqslant 12$, or else $s \geqslant 13$. We divide into cases according to the value of $n$.

Suppose first that $n \leqslant 1$, whence we may assume that $s \geqslant 13$. Then by using the inequality (4.1) once again, we have

$$
\begin{aligned}
f^{h} g^{m} H^{n} & \ll f^{10-n} H^{n}+f^{5-n} g^{5} H^{n} \\
& \ll f^{10}+f^{9} H+f^{5} g^{5}+f^{4} g^{5} H .
\end{aligned}
$$

Consequently, on making use of the trivial bound $F \ll P$, we deduce from (5.2) and (5.3) that

$$
\int_{\mathscr{I}}|\mathscr{F}(\boldsymbol{\alpha}) \mathscr{G}(\boldsymbol{\alpha}) \mathscr{H}(\boldsymbol{\alpha})| d \boldsymbol{\alpha} \ll P^{s-13}\left(\mathscr{I}_{1}+\mathscr{I}_{2}+\mathscr{I}_{3}+\mathscr{I}_{4}\right),
$$

where

$$
\begin{array}{ll}
\mathscr{I}_{1}=\int_{\mathscr{I}} f^{10} F^{3} d \boldsymbol{\alpha}, & \mathscr{I}_{2}=\int_{\mathscr{M}} f^{9} H F^{3} d \boldsymbol{\alpha}, \\
\mathscr{I}_{3}=\int_{\mathscr{M}} f^{5} g^{5} F^{3} d \mathbf{\alpha}, & \mathscr{I}_{4}=\int_{\mathscr{M}} f^{4} g^{5} H F^{3} d \boldsymbol{\alpha} .
\end{array}
$$

But an application of Hölder's inequality, combined with Lemmata 5.1(iv) and 5.2(a), shows that

$$
\mathscr{I}_{1} \ll\left(\int_{\mathbb{H}^{*}} f^{14} d \boldsymbol{\alpha}\right)^{5 / 7}\left(\int_{\mathscr{U}} F^{21 / 2} d \boldsymbol{\alpha}\right)^{2 / 7} \ll\left(P^{9}\right)^{5 / 7}\left(P^{11 / 2} Y^{-\sigma}\right)^{2 / 7} \ll P^{8} Y^{-\tau},
$$

for some positive number $\tau$. Meanwhile, by applying Hölder's inequality in combination with Lemmata 5.1(iv) and 5.2(a), (b), we obtain

$$
\begin{aligned}
\mathscr{F}_{2} & \ll\left(\int_{\mathscr{H} 2} f^{14} d \boldsymbol{\alpha}\right)^{9 / 14}\left(\int_{\mathscr{H}} F^{7} H^{14 / 3} d \boldsymbol{\alpha}\right)^{3 / 14}\left(\int_{\mathscr{H}} F^{21 / 2} d \boldsymbol{\alpha}\right)^{1 / 7} \\
& \ll\left(P^{9}\right)^{9 / 14}\left(P^{20 / 3} Y^{-\sigma}\right)^{3 / 14}\left(P^{11 / 2} Y^{-\sigma}\right)^{1 / 7} \\
& \ll P^{8} Y^{-\tau},
\end{aligned}
$$

for some positive number $\tau$. Next applying Hölder's inequality together with Lemmata 5.1(iii), (iv) and 5.2(a), we deduce that

$$
\begin{aligned}
& \mathscr{I}_{3} \ll\left(\int_{\mathbb{Z}^{*}} f^{6} g^{8} d \boldsymbol{\alpha}\right)^{5 / 8}\left(\int_{\mathbb{R}^{*}} f^{14} d \boldsymbol{\alpha}\right)^{5 / 56}\left(\int_{\mathscr{M}} F^{21 / 2} d \boldsymbol{\alpha}\right)^{2 / 7} \\
& \ll\left(P^{9}\right)^{5 / 8}\left(P^{9}\right)^{5 / 56}\left(P^{11 / 2} Y^{-\sigma}\right)^{2 / 7} \\
& \ll P^{8} Y^{-\tau} \text {, }
\end{aligned}
$$


for some positive number $\tau$. Finally, an application of Hölder's inequality together with Lemmata 5.1(iii), (iv) and 5.2(a), (b) reveals that

$$
\begin{aligned}
\mathscr{I}_{4} & \ll\left(\int_{\mathscr{U}_{2}^{*}} f^{6} g^{8} d \boldsymbol{\alpha}\right)^{5 / 8}\left(\int_{\mathscr{L ^ { * }}} f^{14} d \boldsymbol{\alpha}\right)^{1 / 56}\left(\int_{\mathscr{M}} F^{7} H^{14 / 3} d \boldsymbol{\alpha}\right)^{3 / 14}\left(\int_{\mathscr{H}} F^{21 / 2} d \boldsymbol{\alpha}\right)^{1 / 7} \\
& \ll\left(P^{9}\right)^{5 / 8}\left(P^{9}\right)^{1 / 56}\left(P^{20 / 3} Y^{-\sigma}\right)^{3 / 14}\left(P^{11 / 2} Y^{-\sigma}\right)^{1 / 7} \\
& \ll P^{8} Y^{-\tau},
\end{aligned}
$$

for some positive number $\tau$. Thus, collecting together (5.4)-(5.8), we obtain

$$
\int_{\mathscr{M}}|\mathscr{F}(\boldsymbol{\alpha}) \mathscr{G}(\boldsymbol{\alpha}) \mathscr{H}(\boldsymbol{\alpha})| d \boldsymbol{\alpha} \ll P^{s-5} Y^{-\tau},
$$

for some positive number $\tau$.

Next we suppose that $s \geqslant 12$ and $2 \leqslant n \leqslant 3$. Applying the inequality (4.1) again, we obtain on this occasion

$$
f^{h} g^{m} H^{n} \ll f^{8} H^{2}+f^{7} H^{3}+f^{3} g^{5} H^{2}+f^{2} g^{5} H^{3} .
$$

Thus, again making use of the trivial bound $F \ll P$, we deduce from (5.2) and (5.10) that

$$
\int_{\mathscr{H}}|\mathscr{F}(\boldsymbol{\alpha}) \mathscr{G}(\boldsymbol{\alpha}) \mathscr{H}(\boldsymbol{\alpha})| d \boldsymbol{\alpha} \ll P^{s-12}\left(\mathscr{I}_{5}+\mathscr{I}_{6}+\mathscr{I}_{7}+\mathscr{I}_{8}\right)
$$

where

$$
\begin{array}{ll}
\mathscr{I}_{5}=\int_{\mathscr{M}} f^{8} H^{2} F^{2} d \boldsymbol{\alpha}, & \mathscr{I}_{6}=\int_{\mathscr{M}} f^{7} H^{3} F^{2} d \mathbf{\alpha}, \\
\mathscr{I}_{7}=\int_{\mathscr{M}} f^{3} g^{5} H^{2} F^{2} d \boldsymbol{\alpha}, & \mathscr{I}_{8}=\int_{\mathscr{M}} f^{2} g^{5} H^{3} F^{2} d \boldsymbol{\alpha} .
\end{array}
$$

Applying Hölder's inequality in combination with Lemmata 5.1(i), (iv) and 5.2(a) yields

$$
\begin{aligned}
\mathscr{I}_{5} & \ll\left(\int_{\mathscr{U}_{2}^{*}} f^{8} H^{29 / 7} d \boldsymbol{\alpha}\right)^{14 / 29}\left(\int_{\mathcal{H}^{*}} f^{14} d \boldsymbol{\alpha}\right)^{60 / 203}\left(\int_{\| /} F^{406 / 45} d \boldsymbol{\alpha}\right)^{45 / 203} \\
& \ll\left(P^{50 / 7}\right)^{14 / 29}\left(P^{9}\right)^{60 / 203}\left(P^{181 / 45} Y^{-\sigma}\right)^{45 / 203} \\
& \ll P^{7} Y^{-\tau},
\end{aligned}
$$


for some positive number $\tau$. Also, combining Hölder's inequality with Lemmata 5.1(i), (iv) and 5.2(a), we deduce that

$$
\begin{aligned}
& \mathscr{I}_{6} \ll\left(\int_{\mathbb{H}^{*}} f^{8} H^{9 / 2} d \boldsymbol{\alpha}\right)^{2 / 3}\left(\int_{\mathbb{H}^{*}} f^{14} d \boldsymbol{\alpha}\right)^{5 / 42}\left(\int_{\mathscr{M}} F^{28 / 3} d \boldsymbol{\alpha}\right)^{3 / 14} \\
& \ll\left(P^{15 / 2}\right)^{2 / 3}\left(P^{9}\right)^{5 / 42}\left(P^{13 / 3} Y^{-\sigma}\right)^{3 / 14} \\
& \ll P^{7} Y^{-\tau} \text {, }
\end{aligned}
$$

for some positive number $\tau$. Further, applying Hölder's inequality together with Lemmata 5.1(i), (ii), (iii) and 5.2(a), we obtain

$$
\begin{aligned}
\mathscr{I}_{7} & \ll\left(\int_{\psi^{*}} f^{6} g^{8} d \alpha\right)^{23 / 80}\left(\int_{\mu^{*}} f^{8} H^{640 / 159} d \alpha\right)^{51 / 320}\left(\int_{\mu^{*}} g^{8} H^{640 / 159} d \alpha\right)^{27 / 80}\left(\int_{\| \prime} F^{640 / 69} d \alpha\right)^{69 / 320} \\
& \ll\left(P^{9}\right)^{23 / 80}\left(P^{1117 / 159}\right)^{51 / 320}\left(P^{1117 / 159}\right)^{27 / 80}\left(P^{295 / 69} Y^{-\sigma}\right)^{69 / 320} \\
& \ll P^{7} Y^{-\tau},
\end{aligned}
$$

for some positive number $\tau$. A final application of Hölder's inequality together with Lemmata 5.1(i), (ii), (iii) and 5.2(a) yields

$$
\begin{aligned}
& \mathscr{I}_{8} \ll\left(\int_{\mu^{*}} f^{6} g^{8} d \boldsymbol{\alpha}\right)^{1 / 9}\left(\int_{\mu^{*}} f^{8} H^{216 / 49} d \boldsymbol{\alpha}\right)^{1 / 6}\left(\int_{\mathscr{K}^{*}} g^{8} H^{216 / 49} d \boldsymbol{\alpha}\right)^{37 / 72}\left(\int_{/ /} F^{48 / 5} d \boldsymbol{\alpha}\right)^{5 / 24} \\
& \ll\left(P^{9}\right)^{1 / 9}\left(P^{363 / 49}\right)^{1 / 6}\left(P^{363 / 49}\right)^{37 / 72}\left(P^{23 / 5} Y^{-\sigma}\right)^{5 / 24} \\
& \ll P^{7} Y^{-\tau},
\end{aligned}
$$

for some positive number $\tau$. On collecting together (5.11)--(5.15), therefore, we conclude that the inequality (5.9) holds also in this case.

In order to complete the proof of the lemma, we have only to observe that by Lemma 4.2 and (5.9) we have

$$
\begin{aligned}
\int_{\mathscr{H}^{*}}|\mathscr{F}(\boldsymbol{\alpha}) \mathscr{G}(\boldsymbol{\alpha}) \mathscr{H}(\boldsymbol{\alpha})| d \boldsymbol{\alpha} & =\int_{m}|\mathscr{F}(\boldsymbol{\alpha}) \mathscr{G}(\boldsymbol{\alpha}) \mathscr{H}(\boldsymbol{\alpha})| d \boldsymbol{\alpha}+\int_{\mathfrak{M}}|\widetilde{F}(\boldsymbol{\alpha}) \mathscr{G}(\boldsymbol{\alpha}) \mathscr{H}(\boldsymbol{\alpha})| d \boldsymbol{\alpha} \\
& \ll P^{s-5},
\end{aligned}
$$

and similarly, for some positive number $\tau$,

$$
\begin{aligned}
\int_{1}|\mathscr{F}(\boldsymbol{\alpha}) \mathscr{G}(\boldsymbol{\alpha}) \mathscr{H}(\boldsymbol{\alpha})| d \boldsymbol{\alpha} & =\int_{\mathscr{M} \backslash \mathscr{R}}|\mathscr{F}(\boldsymbol{\alpha}) \mathscr{G}(\boldsymbol{\alpha}) \mathscr{H}(\boldsymbol{\alpha})| d \boldsymbol{\alpha}+\int_{\mathfrak{m}}|\mathscr{F}(\boldsymbol{\alpha}) \mathscr{G}(\boldsymbol{\alpha}) \mathscr{H}(\boldsymbol{\alpha})| d \boldsymbol{\alpha} \\
& \ll P^{s-5} W^{-\tau}+P^{s-5-\delta} \\
& \ll P^{s-5} W^{-\tau} .
\end{aligned}
$$


The end-game analysis for the pruned major arcs $\mathfrak{N}$ is routine, and is discussed in detail in [11, \$10]. On combining the conclusions of Lemmata $10.1,10.3$ and 10.7 of [11], one finds that whenever $s \geqslant 12$ and $W \leqslant R$, one has

$$
\int_{\mathfrak{n}} \mathscr{F}(\boldsymbol{\alpha}) \mathscr{G}(\boldsymbol{\alpha}) \mathscr{H}(\boldsymbol{\alpha}) d \boldsymbol{\alpha}=\boldsymbol{\Xi} \mathscr{C} P^{s-5}+O\left(P^{s-5}\left(W^{4} / \log P+W^{-\tau}\right)\right),
$$

for some $\tau>0$, where $\mathscr{C}$ is a positive constant, and

$$
\Xi=\sum_{q=1}^{\infty} \sum_{a=1}^{q} \sum_{\substack{b=1 \\(a, b, q)=1}}^{q} \prod_{i=1}^{s}\left(q^{-1} S_{i}(q, a, b)\right)
$$

in which we write

$$
S_{i}(q, a, b)=\sum_{r=1}^{q} e\left(\left(c_{i} a r^{3}+d_{i} b r^{2}\right) / q\right) \quad(1 \leqslant i \leqslant s) .
$$

Here we note a misprint in [11]. On page 194, the expression $\lim _{\lambda \rightarrow \infty}$ $\int_{-\lambda}^{\lambda} V(t) e(t \omega) d \omega=V(0)$ should read $\lim _{\lambda \rightarrow \infty} \int_{-T}^{T} \int_{-\lambda}^{\lambda} V(t) e(t \omega) d \omega d t=V(0)$. Moreover, on inserting our assumption that for each prime $p$ one has $M_{n}\left(p^{t}\right) \geqslant p^{(t-u)(s-2)}$, for some $u=u(p)<\infty$, into the conclusions of Lemmata 10.8 and 10.9 of [11], we find that $\Xi>0$. Consequently, on combining (5.16) with the conclusion of Lemma 5.3, and taking $W$ to be a sufficiently small power of $\log P$, we deduce from (3.1) that $R(P) \gg P^{s-5}$, whence Theorem 1 follows immediately.

Acknowledgement. This paper was completed while the author was enjoying the hospitality of the Department of Mathematics at Princeton University: he thanks the U.S. NSF for a grant, and the David and Lucile Packard Foundation for valuable support.

\section{References}

1. R. C. Baker and J. Brüdern. On pairs of additive cubic equations. J. Reine Angew. Math., 391 (1988), 157-180.

2. J. Brüdern. On pairs of diagonal cubic forms. Proc. London Math. Soc. (3), 61 (1990), 273 343.

3. J. Brüdern. On Waring's problem for fifth powers and some related topics. Proc. London Math. Soc. (3), 61 (1990), 457-479.

4. J. Brüdern and R. J. Cook. On simultaneous diagonal equations and inequalities. Acta Arith., 62 (1992), 125-149.

5. L.-K. Hua. Additive Theory of Prime Numbers (American Mathematical Society, Providence, Rhode Island, 1965).

6. R. C. Vaughan. On Waring's problem for cubes. J. Reine Angew. Math., 365 (1986), 122 170.

7. R. C. Vaughan. On Waring's problem for smaller exponents. Proc. London Math. Soc. (3), 52 (1986), 445-463.

8. R. C. Vaughan. A new iterative method in Waring's problem. Acta Math., 162 (1989), 1-71.

9. R. C. Vaughan. The Hardy-Littlewood Method, 2nd Edition, Cambridge Tract No. 125 (Cambridge University Press, Cambridge, 1997).

10. T. D. Wooley. On simultaneous additive equations, I. Proc. London Math. Soc. (3), 63 (1991), $1-34$.

11. T. D. Wooley. On simultaneous additive equations, II. J. Reine Angew. Math., 419 (1991), $141-198$.

12. T. D. Wooley. Large improvements in Waring's problem. Ann. of Math., 135 (1992), 131-. 164. 
13. T. D. Wooley. A note on symmetric diagonal equations. Number Theory with an emphasis on the Markoff Spectrum. A. D. Pollington and W. Moran, eds. (Marcel Dekker, 1993), 317321 .

14. T. D. Wooley. An affine slicing approach to certain paucity problems. Analytic Number Theory: Proceedings of a Conference in Honor of Heini Halberstam. B. C. Berndt, H. G. Diamond and A. J. Hildebrand, ed., vol. 2 (Birkhäuser Boston, Boston, MA, 1996), 803-815.

15. T. D. Wooley. On exponential sums over smooth numbers. J. Reine Angew. Math., 488 (1997), $79-140$.

Professor T. D. Wooley,

Department of Mathematics,

University of Michigan,

East Hall, 525 East University Avenue,

Ann Arbor, MI 48109-1109,

U.S.A.
11D72: NUMBER THEORY; Diophantine Equations; Equations in many variables.

Received on the 3rd of November, 1997. 